\title{
Low frequency seismic noise acquisition and analysis with tunable monolithic horizontal sensors
}

\author{
Fausto Acernese ${ }^{a, b}$, Rosario De Rosa ${ }^{b, c}$, Riccardo De Salvo ${ }^{d}$, Gerardo Giordano $^{a}$, Jan Harms ${ }^{e}$, \\ Vuk Mandic $^{e}$, Rocco Romano ${ }^{a, b}$, Thomas Trancynger ${ }^{f}$, Silvia Vilasi $^{a, b}$, Fabrizio Barone $^{a, b}$ \\ ${ }^{a}$ Università degli Studi di Salerno, Fisciano (SA), Italia \\ ${ }^{b}$ INFN sez. di Napoli, Napoli, Italia \\ ${ }^{c}$ Università di Napoli "Federico II", Napoli, Italia \\ ${ }^{d}$ California Institute of Technology, Pasadena, CA, U.S.A. \\ ${ }^{e}$ University of Minnesota, Twin Cities, MN, U.S.A. \\ $f$ South Dakota Science and Technology Authority, Lead, SD, U.S.A.
}

\begin{abstract}
In this paper we present and discuss the scientific data recorded along one month of data taking of two mechanical monolithic horizontal sensor prototypes located in a blind-ended (side) tunnel $2000 \mathrm{ft}$ deep in the Homestake mine (South Dakota, USA), chosen to host the Deep Underground Science and Engineering Laboratory (DUSEL). The main goal of this test is to provide preliminary data to characterize the Homestake site in the frequency band $10^{-4} \div 30 \mathrm{~Hz}$ and to estimate the level of Newtonian noise, information necessary to understand the feasibility of underground gravitational-wave interferometers sensitive at $1 \mathrm{~Hz}$ and below. The recorded scientific data and all the technical information obtained with this test are very useful also to understand the performances of the monolithic sensors in the very low frequency band $\left(10^{-6} \div 10^{-3} \mathrm{~Hz}\right)$ and to organize an experiment for the low frequency seismic characterization of the Homestake site with monolithic sensors positioned at different levels and orientations.
\end{abstract}

Keywords: Monolithic Sensor, Folded Pendulum, Seismometer, Seismic Noise

\section{INTRODUCTION}

The Homestake mine is the largest known iron-formation-hosted gold-ore body, marked by a rather complicated stratification and history of folding events and metamorphosis of igneous and sedimentary rocks. It lies far from the oceans in the Lead-Deadwood Dome of the Black Hills (South Dakota, USA) and has the deepest reaching tunnels in North America (8000 ft) which provides an optimal stage for monitoring the seismic noise with a three dimensional network of seismometers. In figure1 a picture of one of the admittances to the mine is shown.

Recently, the Homestake mine was chosen to host the Deep Underground Science and Engineering Laboratory (DUSEL). For this task, in August 2008 a first series of seismic measurements were performed at different underground levels in the Homestake mine, the $300 \mathrm{ft}$, the $800 \mathrm{ft}$ and $2000 \mathrm{ft}$ levels, intended as a starting point for a more exhaustive survey. ${ }^{1}$

In December 2008, the group of Applied Physics of the University of Salerno installed at Homestake two prototypes of monolithic seismometers in thermally insulating enclosures onto concrete slabs connected to the bedrock and behind a sound-proofing wall. The main goal of this test is to provide preliminary data necessary to characterize the Homestake site in the frequency band $10^{-4} \div 30 \mathrm{~Hz}$ and to estimate the level of Newtonian noise, information necessary to understand the feasibility of underground gravitational-wave interferometers sensitive at $1 \mathrm{~Hz}$ and below. The recorded scientific data and all the technical information obtained with this test will also be used both to understand the performances of the monolithic sensors in the very low frequency band $\left(10^{-6} \div 10^{-3} \mathrm{~Hz}\right)$ and to organize an experiment for the low frequency seismic characterization of the Homestake site with monolithic sensors positioned at different levels.

Send correspondence to Fabrizio Barone - e-mail: fabrizio.barone@na.infn.it

Earth Resources and Environmental Remote Sensing/GIS Applications, edited by Ulrich Michel, Daniel L. Civco, Proc. of SPIE Vol. 7831, 78311D · C 2010 SPIE · CCC code: 0277-786X/10/\$18 · doi: 10.1117/12.865069 


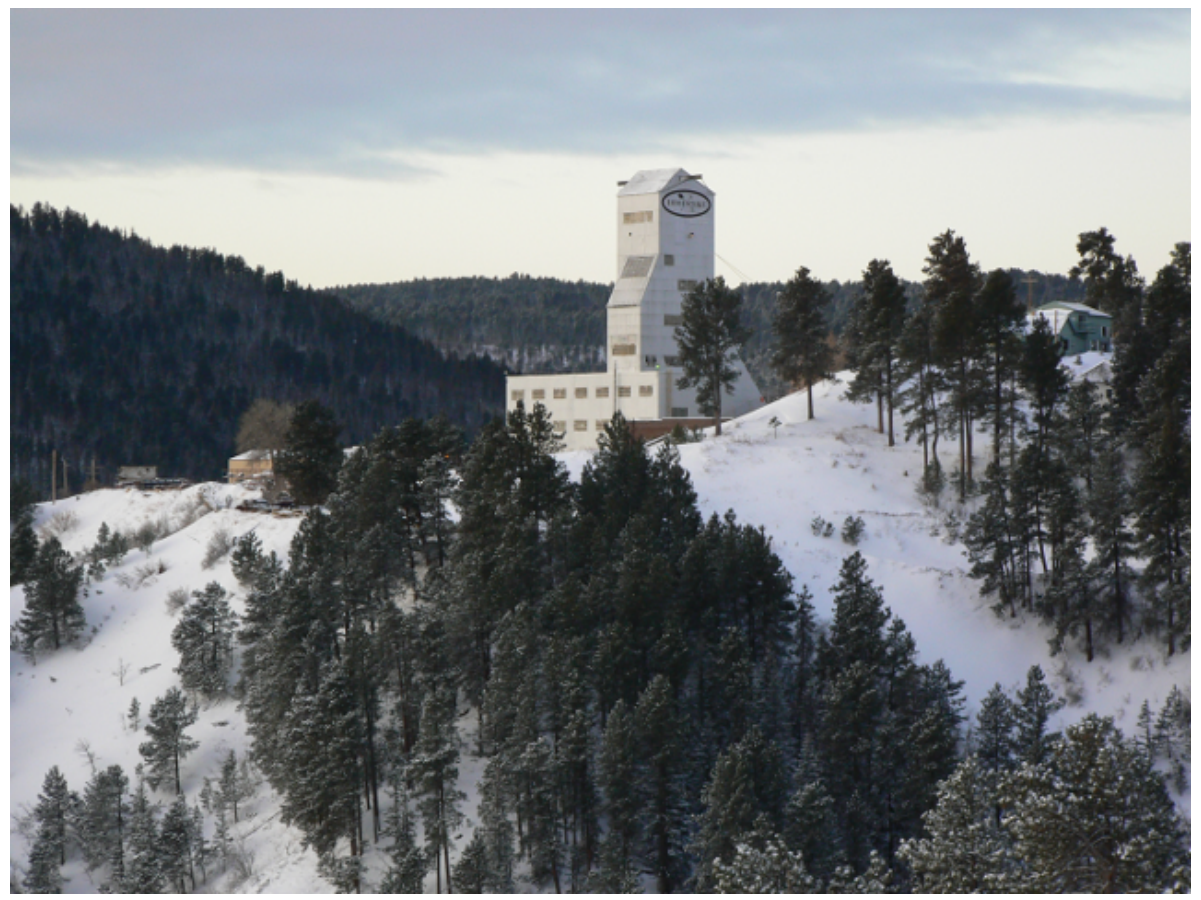

Figure 1. One of the admittances to the Homestake mine

In this paper the tunable monolithic seismic sensor prototypes, the sensors installation at $2000 \mathrm{ft}$ and the architecture of the DAQ system are described. Then, the first scientific data acquired are presented and discussed, together with the expected future development and improvements.

\section{THE MONOLITHIC SENSOR}

The structure of the tunable mechanical horizontal monolithic sensor is based on the Folded Pendulum (hereafter FP), called also Watt-linkage, a classical suspension system developed in $1962,{ }^{2}$ recently redescovered for application in gravitational wave research as ultra-low frequency pendulum resonators for vibration isolation in interferometric detectors of gravitational waves. ${ }^{3}$

Based on the FP basic scheme, single-axis monolithic accelerometers have been developed as sensors in the control system for advanced seismic attenuators. ${ }^{4}$ The progress in precision micro-machining, that has allowed the construction of tunable sensors of small size with extremely soft flexures at the pendulum's hinges, and the innovative application of laser optics readout techniques have largely improved the sensor sensitivity in the low frequency band and increased its immunity to environmental noises. ${ }^{5,6}$ Special tuning procedures have been also developed to decrease the sensor natural frequency (to values as low as $\approx 70 \mathrm{mHz}$ ) and dimensions, allowing its use both as seismometer and accelerometer for seafloors or boreholes. ${ }^{5}$

On the basis of these scientific results, a low-noise high-resolution horizontal monolithic FP sensor was developed at the University of Salerno for geophysical applications aimed to explore the low frequency band of the seismic spectrum, both as a stand-alone sensor or as part of large and geographically distributed seismic networks.

The theoretical FP transfer function, describing the FP dynamics as seismometer and/or accelerometer, can be easily obtained using the simplified Lagrangian model developed by J.Liu et al. ${ }^{3}$ This model, based on the mechanical scheme shown in Figure 2(a), describes only the FP basic dynamics, but it is anyway very useful to understand the FP main characteristics and expected performances.

In particular, defining the coordinate of the pendulum frame (fixed to the ground) as $x_{g}$ and the coordinate of the FP central mass as $x_{c}$ (Figure 2(a)), then the mass displacement transfer function with respect to the 


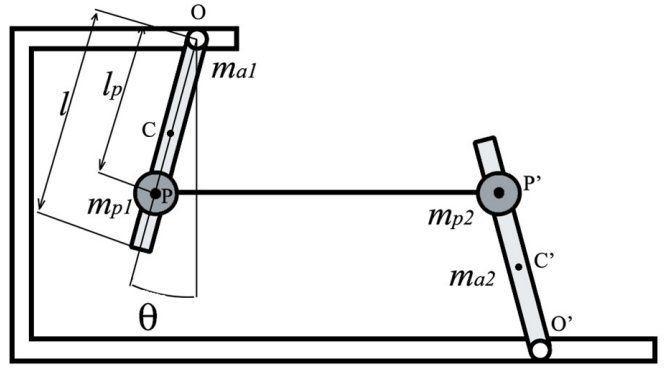

(a) Scheme of Folded Pendulum.

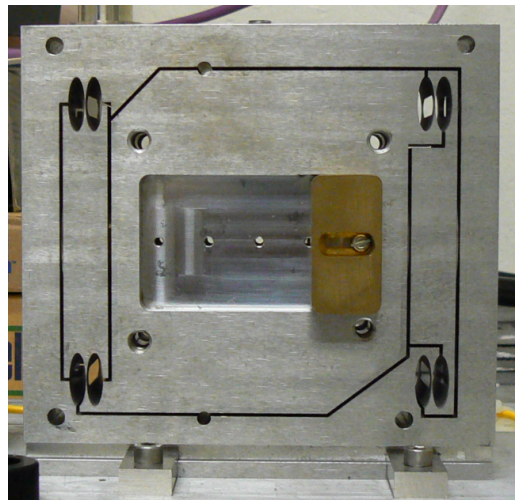

(b) Pictures of the Mechanical monolithic horizontal seismometer prototype (mod. 08F_100_AL1).

ground displacement can be obtained as

$$
\frac{x_{c}(s)-x_{g}(s)}{x_{g}(s)}=\frac{\left(G_{p}-1\right) s^{2}}{s^{2}+\frac{\omega_{o}}{Q\left(\omega_{o}\right)} s+\omega_{o}^{2}}
$$

where $Q\left(\omega_{o}\right)$ is the global quality factor, that includes the dissipative effects,

$$
G_{p}=\frac{\left(\frac{l}{3 l_{p}}-\frac{1}{2}\right)\left(m_{a_{1}}-m_{a_{2}}\right)}{\left(m_{a_{1}}+m_{a_{2}}\right) \frac{l^{2}}{3 l_{p}^{2}}+\left(m_{p_{1}}+m_{p_{2}}\right)}
$$

is the parameter related to the center of percussion effects, ${ }^{3}$ and, named $k$ the cumulative angular spring constant,

$$
\omega_{o}=\sqrt{\frac{\left(m_{a 1}-m_{a 2}\right) \frac{g l}{2 l_{p}^{2}}+\left(m_{p 1}-m_{p 2}\right) \frac{g}{l_{p}}+\frac{k_{\theta}}{l_{p}^{2}}}{\left(m_{a 1}+m_{a 2}\right) \frac{l^{2}}{3 l_{p}^{2}}+\left(m_{p 1}+m_{p 2}\right)}}
$$

is the FP angular resonance frequency. The configuration of the monolithic FP as seismometer is straightforward: the readout output signal $\left(x_{p}(\omega)-x_{s}(\omega)\right)$ is acquired, calibrated and processed according to Equation 1.

The sensor prototype (mod. 08F_100_AL1) is made of Aluminium, shaped with precision machining and electric discharge machining (EDM) from a $134 \times 134 \times 40 \mathrm{~mm}$ block of metal (Alloy 7075-T6) (Figure 2(b)). The four flex joints have an elliptical profile with $100 \mu \mathrm{m}$ minimum thickness and ellipticity ratio of $\epsilon=16 / 5$. This shape ensures robustness and long-term durability to the mechanics. ${ }^{5,6}$ The pendula arms $(71.5 \mathrm{~mm}$ length and spaced by $102 \mathrm{~mm}$ ) minimize mass and moment of inertia, without reducing rigidity and symmetry. The values of the masses of the pendulum arm, of the inverted pendulum arm and of the central mass are $m_{p} \approx 40 \mathrm{~g}$, $m_{i p} \approx 50 \mathrm{~g}$ and $m_{c} \approx 600 \mathrm{~g}$, respectively. The FP frequency tunability was obtained as described in, ${ }^{5}$ machining a large recess in the central mass to hosting suitable shaped and positioned tuning masses. The gaps between central mass-arms and arms-frame are $1 \mathrm{~mm}$ large to increase the FP mechanical dynamics and the quality factor for applications in air.

We measured the two sensitivity curves corresponding to optical levers (relized with PSD) and interferometer readouts. In Figure 2 the best theoretical and experimental sensitivities curves at $T=300 \mathrm{~K}$ and $f_{o}=70 \mathrm{mHz}$ in air and without thermal stabilization are shown. To compare the sensitivity of the FP seismometer with other geophysical instruments, we reported in Figure 2 also the sensitivity of the STS-2 by Streckeisen, that represents the state-of-art of the low frequency seismic sensors. ${ }^{7}$ Figure 2 shows that the monolithic FP sensor has already a sensitivity comparable or better (with interferometric readout) than the STS-2 in the band $10^{-3} \div 10 \mathrm{~Hz}$. We reported also the Peterson New Low Noise Model (NLNM), ${ }^{8}$ that is the Minimum Earth Noise evaluated from a collection of seismic data from 75 sites located around the world, that describes the minimum level of earth noise: noise levels below this are never - or extremely rarely - observed. 


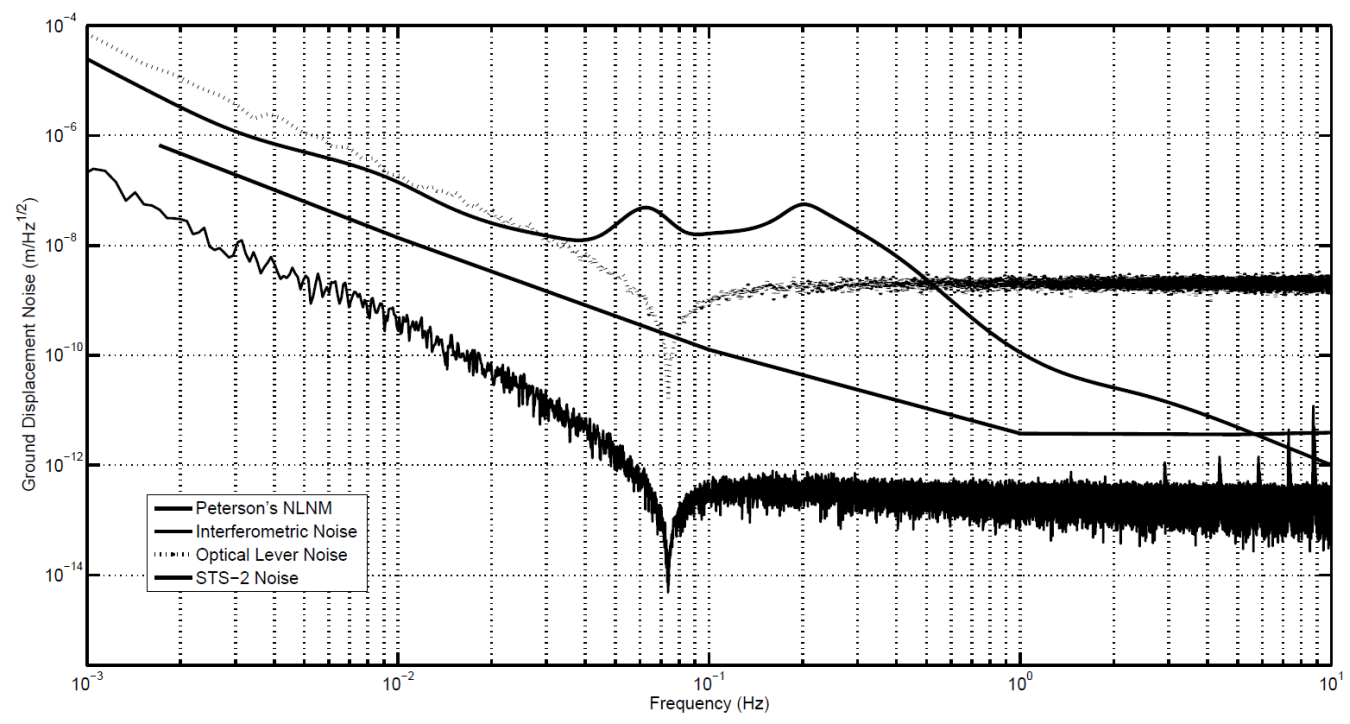

Figure 2. Theoretical and experimental sensitivity curves of the monolithic FP seismometer.

\section{THE $2000 F T$ SEISMIC STATION AT HOMESTAKE}

The $2000 \mathrm{ft}$ station was built in August 2008 on the concrete platform of an old charging station located on the bedrock, having carefully checked that any audible source of dropping water were eliminated as good as possible. It was obtained sectioning a tunnel and sealing it from two sides with double layered walls. Many instruments were located in the station inside a specially designed insulated box. A separate hut at some distance to the instrument area hosts the data acquisition PCs and the DC power supply. ${ }^{1}$

The environmental conditions at the $2000 \mathrm{ft}$ level are stable around $20^{\circ} \mathrm{C}$ with an rms of about $0.2^{\circ} \mathrm{C}$. Sensors are installed close to the seismometers to monitor the most important environmental quantities: temperature, pressure, humidity, sound and magnetic field. Humidity monitoring is relevant because the humidity levels may reach $100 \%$ and condensation of water on the electronics could start soon, although the insulation boxes are good enough to bring humidity down to $80 \%-90 \%$ also in presence of $100 \%$ humidity. Pressure and sound monitoring is important because these quantities may show significant correlations with seismic data since both could generate rock vibrations or may even directly act on seismometers despite all the efforts to isolate the instruments.

The data-acquisition system includes the configuration of the local network and remote access to PCs. The acquisition system is based on the PCI 6289 card from National Instruments ${ }^{\mathrm{TM}}$. It contains a 18 bit ADC and an internal amplifier with a maximum amplification of 100. The input range is limited to $\pm 10 \mathrm{~V}$. The Data Acquisition is managed by Labview ${ }^{\mathrm{TM}}$ programs which also generate analog-output signals to initiate masscentering procedures of seismometers, if necessary. The data are first stored in ASCII files as $128 s$ records sampled at $100 \mathrm{~Hz}$. Then the data is made available to the outside world by an ftp server while the timing signals are provided to the acquisition system through optical fibers.

The sequence of installation of the monolithic sensor within the insulated box of the $2000 \mathrm{ft}$ station is shown in Figure 3(a), 3(b) and 3(c).

\section{EXPERIMENTAL RESULTS}

In this section we present some of the preliminary results of the first month of data acquisition of FP monolithic sensor prototypes. In particular, the power spectral density of ground displacement at $2000 \mathrm{ft}$ underground level (compared with the theoretical expected FP noise and Peterson's New Low Noise Model) is shown in Figure 3. What is relevant to note is its large measurement band $\left(10^{-5} \div 30 \mathrm{~Hz}\right)$ coupled with a good sensitivity, although the latter is largely limited by our choice of using an optical lever as readout and to the safe resonance frequency 


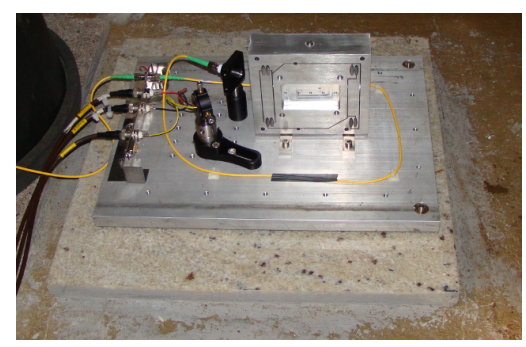

(a) Assembly fo the sensor with optical lever readout.

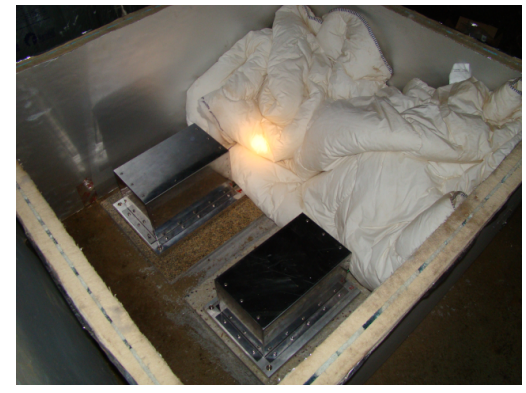

(b) Sensors closed in their boxes and then in a thermal acoustic box. The duvet keeps the ground isothermal and avoid convection.

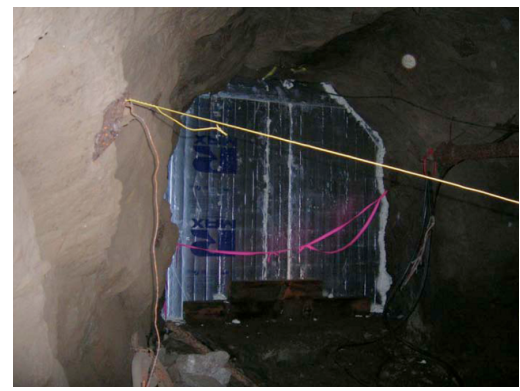

(c) The thermal acoustic hut is finally closed.

chosen for this first test in the Homestake mine $(300 \mathrm{mHz})$. Tuning the FP sensor at a lower resonance frequency would simply translate the sensitivity curve towards the low frequency region, enlarging the low frequency band. Nevertheless, even with this not optimal configuration, the instruments is able to detect part of the Peterson Low Noise Spectrum and, in the very low frequency region, the peaks due to Earth tides (Figure 3).

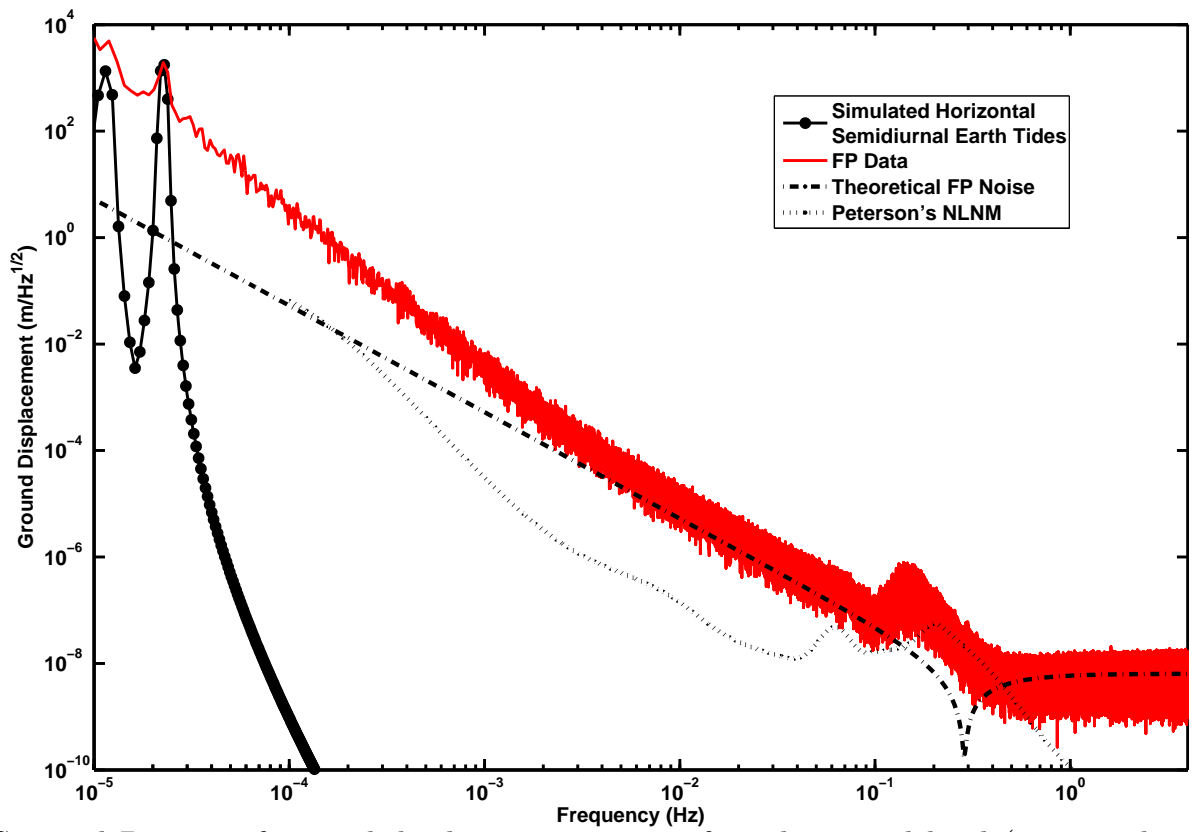

Figure 3. Power Spectral Density of ground displacement at $2000 \mathrm{ft}$ underground level (compared with theoretical FP noise, simulated horizontal semidiurnal Earth tides and Peterson's New Low Noise Model).

The apparent limitation in the low frequency band is simply due to the fact that the power spectral density has been evaluated only for one month of data. Longest runs may allow the exploration of lower frequency regions. In fact, the FP monolithic sensor is an open loop sensor, so that there are no limitations coming from the feed-back control, as it happens in the majority of the commercial instruments used for seismic noise acquisition. The limitations are mainly due to the electronic noise of the readout system, to the thermal noise of the mechanical joints and to the effects of the air for sensors not operating in vacuum.

For completeness, the time behavior and the spectrogram of about 20 days of data of ground displacement as recorded by our seismometer is shown in Figure 4. As it can been seen the resonant frequency is very stable and the instrument is able to measure teleseimic earthquake. A longer acquisition time is necessary for a more accurate analysis of its performances, even if the preliminary results are very promising. 


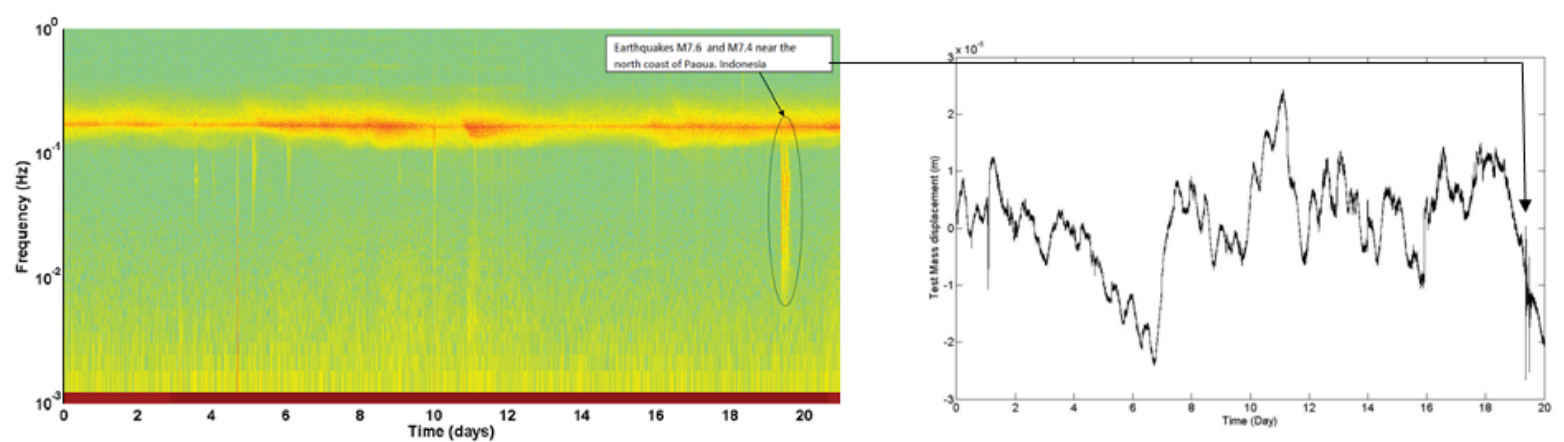

Figure 4. the Spectrogram (on the left) and the time behaviour (on the right) of about $500 h$ of ground displacement, as recorded by our seismometer. Two teleseismic earthquakes near north coast of Papua, Indonesia, are shown (January, 03, 2009, 19:43:50 UTC time and Saturday, January 03, 2009, 22:33:40 UTC time).

\section{CONCLUSIONS AND FUTURE DEVELOPMENTS}

Two FP monolithic sensors are installed into the $2000 \mathrm{ft}$ station in the Homestake mine since December 2008, equipped with a National Instruments ${ }^{\mathrm{TM}}$ (32 channels - 18 bit ADC) data acquisition system, a network access through optical fibre cables and an environmental monitoring system.

The FP sensor sensitivity is close to the theoretical one in the band $\left(10^{-5} \div 30 \mathrm{~Hz}\right)$ at the resonance frequency of about $300 \mathrm{mHz}$ for the optical lever readout configuration. Better sensitivities could be obtained reducing the resonance frequency of the instrument and using more sensitive optical readouts, but it was preferred to priviledge the instrument robustness for this first unattended measurement in the Homestake mine. Nevertheless, even with this not optimal configuration, the instruments are able to detect part of the Peterson Low Noise Spectrum and, in the very low frequency region, the peaks due to Earth tides.

The recorded scientific data and all the technical information obtained with this test are very useful also to understand the performances of the monolithic sensor in the very low frequency band $\left(10^{-6} \div 10^{-3} \mathrm{~Hz}\right)$. This information will be used to organize an experiment for the low frequency seismic characterization of the Homestake site with an improved version of these sensors to be positioned at different levels and orientations.

\section{Acknowledgment}

We acknowledge Galli \& Morelli Factory and, in particular, Mr. Carlo Galli for the useful suggestions and for the technical support in the development and implementation of mechanical part of the monolithic FP sensor.

This work is part of the PRIN 2007 Research Project Low frequency sensitivity in underground cryogenic gravitational wave interferometers (Project 2007NXMBHP), partly supported by the Ministero dell'Istruzione, dell'Università e della Ricerca (MIUR), Italy.

\section{REFERENCES}

[1] F. Acernese, R. DeSalvo, J. Harms, A. Sajeva, L. Shaffner, T. Trancynger, LIGO T080344-00-R, Caltech, USA (2008).

[2] E. S. Fergusson, US Nat. Museum Bull., 228, 185 (1962).

[3] J. Liu, L. Ju, D.G. Blair, Phys. Lett. A, 228, 243-249 (1997).

[4] A. Bertolini et al., Nucl. Instr. and Meth., 556, 616-623 (2006).

[5] F. Acernese, R. De Rosa, G. Giordano, R. Romano, F. Barone, Rev. Sci. Instrum., 79, 074501, doi:10.1063/1.2943415 (2008).

[6] F. Acernese, R. De Rosa, G. Giordano, R. Romano, F. Barone, Proc. SPIE Vol. 7292, SPIE, Bellingham, WA, 69320K, 72922J, doi: 10.1117/12.814106 (2009).

[7] Y. Nakayama et al., Proc. of IWAA2004, CERN, Geneva, 4-7 October 2004.

[8] J. Berger, P. Davis, 2005 IRIS 5-Year Proposal, 38 (2005). 